\title{
O PAPEL DO REGANHO DE PESO NO CONTEXTO DA CIRURGIA BARIÁTRICA E NO CONTROLE DE DOENÇAS METABÓLICAS ASSOCIADAS
}

\author{
THE ROLE OF WEIGHT REGAIN IN THE CONTEXT OF BARIATRIC SURGERY AND IN THE CONTROL \\ OF ASSOCIATED METABOLIC DISEASES
}

Mayara Ribeiro Wobido, Daniel Valões Dytz, Rodrigo Chaves Teixeira, Giulia Santos Pignata, Olga de Castro Dytz e Márcio Garrison Dytz

DOI - 10.5935/2236-5117.2015v52n3/4a06

\section{RESUMO}

A obesidade é considerada um problema mundial de agravo à saúde, de causa complexa, e inclui uma combinação de influências psicossociais, ambientais e biológicas. A cirurgia bariátrica é tratamento mais eficaz para pacientes com obesidade grave, resultando na melhora do quadro das comorbidades relacionadas à obesidade. No entanto, o reganho de peso e a recidiva de doenças metabólicas, como diabetes e hipertensão, têm sido observados em um subgrupo de pacientes pós-cirurgia bariátrica. 0 manejo por equipe multidisciplinar com avaliações periódicas é fundamental para otimizar os resultados obtidos desses pacientes.

Palavras-chave. Cirurgia bariátrica; obesidade; diabetes mellitus; reganho de peso

\section{ABSTRACT}

Obesity is considered a worldwide major health problem with complex causes and includes a combination of psychosocial, enviromental and biological aspects. Bariatric surgery is the most eficient treatment for patients with morbid obesity, resulting in improvement of the aspects on obesity-related comorbidities. However, weight regain and recurrence of metabolic disorders such as diabetes and hypertesion have been observed in a subgroup of patients after bariatric surgery. Management by a multidisciplinary team with periodic evaluations is fundamental to optimize the results obtained from these patients.

Key words. Bariatric surgery; obesity; diabetes mellitus; weight regain

\section{INTRODUÇÃO}

A obesidade é uma condição inflamatória crônica, de caráter epidêmico, com impacto negativo na qualidade de vida do indivíduo e em âmbito de saúde pública, uma vez que altera o status metabólico do acometido, podendo provocar surgimento e agravamento de comorbidades. ${ }^{1}$ É condição marcada
Mayara Ribeiro Wobido - acadêmica de medicina, Centro Universitário de Brasilia (UniCEUB)

Daniel Valões Dytz - acadêmico de medicina, Centro Universitário de Brasília (UniCEUB)

Rodrigo Chaves Teixeira - acadêmico de medicina, Centro Universitário de Brasilia (UniCEUB)

Giulia Santos Pignata - acadêmica de medicina, Centro Universitário de Brasília (UniCEUB)

Olga de Castro Dytz - médica endocrinologista, mestre em medicina pela Universidade Federal do Rio de Janeiro (UFRJ), doutoranda em medicina pela Universidade de Brasília (UnB)

Márcio Garrison Dytz - médico endocrinologista, doutor em medicina pela Universidade Federal do Rio de Janeiro (UFRJ), supervisor da residência médica de Endocrinologia e Metabologia Integrada da Secretaria de Estado de Saúde (SES-DF), professor em medicina pelo Centro Universitário de Brasília (UniCEUB), vice-presidente da Sociedade Brasileira em Endocrinologia e Metabologia regional DF (SBEM-DF)

Correspondência: Márcio Garrison Dytz. IDEB -SHS quadra 6, conjunto A, bloco E, sala n. ${ }^{\circ} 1.119$, CEP 70316-902, Brasília-DF.

Telefone: (61) 3039-8439.

Internet: marcio.dytz@uniceub.br

Conflito de interesses: os autores declaram inexistência de conflito de interesses.

Suporte. Os autores agradecem o apoio do CNPq no desenvolvimento deste trabalho, através da concessão de bolsa de pesquisa.

por acúmulo excessivo de gordura central, associado a desproporção entre peso e altura, que acarreta prejuízos à saúde dos indivíduos. ${ }^{2,3}$

As evidências atuais de estudos epidemiológicos de grande e longo prazo indicam que o excesso de peso está associado a incremento da taxa de mortalidade e pode reduzir a expectativa de vida em cerca de cinco a vinte anos, a depender da etnia, da idade e do sexo dos doentes. ${ }^{4-7}$ 
Os estudos de previsão quanto à prevalência de obesidade em adultos variam de $42 \%$ a $51 \%$. Entretanto, é inquestionável que a taxa de prevalência da obesidade aumentou na maior parte do mundo ao longo dos últimos trinta anos. Em plano geral, estimase que mais de $20 \%$ da população mundial encontrase com sobrepeso e aproximadamente 10\% são considerados obesos. ${ }^{8-10}$

No Brasil, sabe-se que o sobrepeso é uma questão de saúde pública, presente em $54 \%$ da população, e para obesos, essa frequência encontra-se em torno de $18 \%$. O Distrito Federal apresenta as menores frequências tanto em questão ao sobrepeso quanto aos obesos, com $47,6 \%$ e $15,3 \%$ de prevalência respectivamente. Além disso, é importante ressaltar que sua prevalência aumenta em grupos com menor grau de escolaridade. ${ }^{11}$

Quanto à causa, apesar de desconhecida até então, acredita-se ser combinação de influências psicossociais - como status socioeconômico, fatores culturais, iatrogênicos, ambientais e genéticos. ${ }^{12,13}$ Vale destacar que a obesidade é uma condição muito relacionada com hábitos de vida, como sedentarismo e uso de dietas ricas em carboidratos e lipídios. ${ }^{3}$

A obesidade apresenta um forte componente hereditário, com uma herança poligênica, a qual conta com vários genes que interagem para determinar essa condição. O fator genético pode contribuir, em até $70 \%$ dos casos de ganho de peso, para induzir maior sensação de fome, menor saciedade, preferência por alimentos hipercalóricos, compulsão alimentar e outras alterações. ${ }^{12,13} \mathrm{Na}$ maioria dos casos, a obesidade é consequência de ambientes obesogênicos, os quais afetam indivíduos geneticamente predispostos.

A condição também ganha importância por ser fator de risco digno de nota relacionados a distúrbios como diabetes melito do tipo 2, hipertensão arterial sistêmica, doenças cardíacas, síndrome da apneia obstrutiva do sono, asma, doença hepática gordurosa não alcoólica, função pulmonar reduzida e aumento do risco de neoplasias malignas. ${ }^{2,14}$

Sua mensuração é realizada por meio de medidas antropométricas sobretudo do índice de massa corporal (IMC). Classifica-se o paciente em sobrepeso caso o IMC seja entre 25 e $29,9 \mathrm{~kg} / \mathrm{m}^{2}$, e obesidade se for maior ou igual a $30 \mathrm{~kg} / \mathrm{m}^{2}$. 3

Por sua importância mundial foram empreendidos esforços ao longo dos anos para estabelecer a terapêutica adequada para controle dessa condição. Recorre-se a dois tipos de conduta, isto é, manejo clínico ou conservador, ou cirúrgico. Inicialmente, opta-se por conduta conservadora. Caso esta não seja eficaz, indica-se cirurgia bariátrica. ${ }^{13}$

Como conduta clínica, inicialmente, são oferecidas orientações quanto a terapia cognitivocomportamental, bem como adoção de prática esportiva feita com regularidade e, sobretudo, modificações dietéticas com base na restrição calórica para induzir balanço energético negativo..$^{15}$ Caso isso não seja eficaz e houver permanência do índice de massa corporal maior ou igual a $30 \mathrm{~kg} / \mathrm{m}^{2}$ ou maior ou igual a $25 \mathrm{~kg} / \mathrm{m}^{2}$ em presença de comorbidades, iniciase tratamento medicamentoso com medicações onlabel, como sibutramina, liraglutida, orlistate ou locarserina. A depender do contexto, recorre-se às drogas off-label, como bupropiona, topiramato ou sertralina como opções químicas. ${ }^{13}$

Apesar de suas indicações, acredita-se que uma grande parcela de pacientes tem resposta insatisfatória à terapêutica medicamentosa em longo prazo devido ao componente crônico e recidivante da obesidade, o que torna a estratégia cirúrgica uma alternativa interessante. ${ }^{16-18}$ Ela é considerada o método mais eficaz para tratamento da obesidade e está reservada para pessoas de 18 a 65 anos, com índice de massa corporal maior ou igual a $40 \mathrm{~kg} / \mathrm{m}^{2}$, ou maior ou igual a $35 \mathrm{~kg} / \mathrm{m}^{2}$ em presença de ao menos uma comorbidade. ${ }^{13,19}$

Evidências atuais apontam a cirurgia bariátrica como o tratamento da obesidade grave mais eficiente se comparado à atividade física, modificações no estilo de vida e medicamentos. Em particular, o procedimento é mais eficaz para alcançar perda de peso significativa, manutenção deste em longo prazo e remissão de distúrbios associados. ${ }^{20,21}$

Com a intervenção cirúrgica em indivíduos obesos, nota-se, além da perda de peso, a remissão de comorbidades - como diabetes melito do tipo 2, hipertensão arterial sistêmica e dislipidemia. Esses achados forneceram subsídios para a criação do termo "cirurgia metabólica", o que destaca o benefício metabólico geral do procedimento, além da perda de peso. 22,23

Para que o procedimento seja considerado satisfatório, deve-se obter perda de $50 \%$ do excesso de peso no período de um ano depois da operação. A manutenção da perda de peso costuma ocorrer até o terceiro ano após a cirurgia bariátrica. Entretanto, isso tende a diminuir com o tempo e estabilizar em 12 a 24 meses após a conduta cirúrgica, com possível reganho depois do segundo ano. Alguns pacientes podem recuperar o peso pré-operatório e apresentar recorrência das comorbidades controladas após a intervenção cirúrgica. ${ }^{24,25}$ 
Algumas fórmulas são utilizadas como ferramentas na análise de eficácia da cirurgia bariátrica, como:

\footnotetext{
Peso ideal $=(\text { altura em } \mathrm{m})^{2} \times 25$

Perda de peso $=$ peso pré-operatório - peso mínimo pós-operatório Percentagem de perda de peso $=($ perda de peso $) /$ (peso préoperatório) x 100

Excesso de peso $=$ peso pré-operatório - peso ideal

Percentagem de perda do excesso de peso $(\% \mathrm{PEP})=($ perda de peso $)$

/ (excesso de peso) $\times 100$

Reganho de peso = peso atual - peso mínimo pós-operatório

Porcentagem de reganho de peso $=($ reganho de peso $) /($ peso mínimo) x 100
}

Segundo a American Heart Association, nos pacientes obesos, a meta é manter a pressão arterial inferior a 130 por $80 \mathrm{mmHg}$. De acordo com o último consenso correlato ao manejo da hipertensão arterial, a redução de peso em pacientes obesos influencia nos valores da pressão arterial, considerando-se a aparente resposta em que a perda de um quilograma de peso corresponda à redução de $1 \mathrm{mmHg}$ no valor da pressão sistólica. ${ }^{26}$

A avaliação da remissão do diabetes, pelo Consenso Americano, é classificada em parcial, completa e prolongada. Remissão parcial é definida como hiperglicemia abaixo do valor diagnóstico do diabetes (hemoglobina glicada não diagnóstica de diabetes [< $6,5 \%$, glicemia de jejum de 100 a $125 \mathrm{mg} / \mathrm{dL}$ ) com duração de pelo menos um ano e ausência de terapia medicamentosa ou de procedimentos em curso; remissão completa é marcada por medida normoglicêmica (hemoglobina glicada dentro da referência normal, glicemia em jejum menor que $100 \mathrm{mg} / \mathrm{dL}$ ) com pelo menos um ano de duração e ausência de farmacoterapia ou de procedimentos em curso; a remissão prolongada, por sua vez, é definida pela remissão completa com pelo menos cinco anos de duração. ${ }^{27}$

A remissão da dislipidemia é definida como a melhora no perfil lipídico do paciente sem uso de medicamentos, com teor de colesterol total menor que $200 \mathrm{mg} / \mathrm{dL}$, $\mathrm{HDL}$ acima de $40 \mathrm{mg} / \mathrm{dL}, \mathrm{LDL}$ abaixo de $160 \mathrm{mg} / \mathrm{dL}$ e teor de triglicerídios abaixo de $200 \mathrm{mg} / \mathrm{dL}$. 0 bypass gástrico em Y de Roux (RYGB) apresenta o melhor efeito sobre a dislipidemia seguido à gastrectomia vertical e, posteriormente, da banda gástrica. ${ }^{25}$

O grupo dos autores do presente trabalho tem estudos em andamento com investigação da eficácia da cirurgia bariátrica nos pacientes operados no Hospital Regional da Asa Norte (HRAN). Os dados coletados são referentes ao período dos anos 2009 até 2018, com mais de mil pacientes captados e avaliação de perda de peso, remissão de doenças e reganho de peso, além da investigação de fatores ambientais, padrão alimentar, atividade física, distúrbios psíquicos e sociais que podem contribuir para o desfecho em longo prazo da cirurgia bariátrica.

0 presente estudo tem por intuito realizar uma revisão bibliográfica a respeito da terapêutica da obesidade, bem como do seguimento de pacientes que foram submetidos à cirurgia bariátrica. Além disso, foram estudados e pesquisados temas associados a esse tema, como comorbidades e fatores que influenciam positivamente ou não no seguimento de pacientes operados.

\section{MÉTODO}

A revisão bibliográfica utilizou bases de dados nacionais e internacionais, como Scielo, EBSCO, Pubmed e Lilacs. Os artigos de referência foram obtidos por meio das palavras-chave obesidade, cirurgia bariátrica, comorbidades, perda de peso, reganho de peso, bypass, remissão. Foram revisados quatrocentos artigos, sendo selecionados 44 para utilização neste estudo.

\section{RESULTADOS}

São recentes os conceitos inerentes ao procedimento cirúrgico a serem utilizados no decorrer do seguimento do paciente submetido à cirurgia bariátrica. Até o ano de 2015, não havia consenso na literatura para definir o sucesso da cirurgia bariátrica e metabólica. A partir do ano citado, a Sociedade Brasileira de Cirurgia Bariátrica se posicionou a respeito desse tema. ${ }^{28}$

No espectro das definições de sucesso ou do insucesso da intervenção cirúrgica, agrupou-se a obesidade em três categorias, a saber, obesidade controlada, parcialmente controlada e não controlada. Instituiu-se como obesidade controlada aqueles pacientes que atingiram perda do peso total acima de $20 \%$ em seis meses; obesidade parcialmente controlada, como perda do peso total de $10 \%$ a $20 \%$ em seis meses; obesidade não controlada, como perda do peso total em menos de $10 \%$ em seis meses. ${ }^{28}$

O conceito de recidiva foi caracterizado como recuperação de $50 \%$ do peso perdido ou recuperação de $20 \%$ do peso, associada ao reaparecimento de comorbidades. A recidiva é dita controlada quando há recuperação de $20 \%$ a $50 \%$ do peso perdido em longo prazo. Por fim, o ganho de peso esperado é caracterizado como recuperação de mais de $20 \%$ do peso perdido em longo prazo. ${ }^{28}$

King e colaboradores ${ }^{29}$ verificaram as diversas formas de avaliação do reganho de peso. Compararam diferentes análises acerca da eficácia 
cirúrgica como ferramenta de perda de peso na obesidade. As seguintes medidas foram comparadas: reganho absoluto do peso maior ou igual a $10 \mathrm{~kg}$; aumento maior ou igual a $5 \mathrm{~kg} / \mathrm{m}^{2}$ do índice de massa corporal; aumento maior ou igual a $10 \%$ do peso préoperatório; aumento maior ou igual a $15 \%$ do peso mínimo; reganho de peso maior ou igual a $10 \%, 20 \%$ e $25 \%$ da perda de peso obtida. Como resultado, obtevese o reganho maior ou igual a $20 \%$ da perda de peso como melhor método para avaliar a eficácia da cirurgia bariátrica.

O reganho de peso é um cenário frequente entre os pacientes pós-bariátrica. Os principais fatores associados são de ordem socioeconômica, além de sedentarismo, distúrbios alimentares, alterações hormonais e metabólicas e comorbidades psiquiátricas como ansiedade e depressão.

Por sua relevância, muito se tem discutido a respeito dos motivos que podem estar relacionados com a recuperação do peso depois do procedimento. No estudo LABS2, de Belle e colaboradores, obtevese uma amostra de 2.458 pacientes e destaca-se a importância do perfil biopsicossocial do paciente obeso. Detectou-se que é quase regra a associação de comorbidades, 95\% no caso da coorte em questão, cujo impacto na qualidade de vida é relevante a ponto de causar prejuízos à autoestima e à vida sexual dos indivíduos, o que acarreta repercussões de ordem psíquicas. ${ }^{30}$

Nedelcu e colaboradores discutiram o conceito de reganho de peso baseado nas seguintes definições: ganho absoluto de $10 \mathrm{~kg}$ com relação ao menor peso atingido e aumento do índice de massa corporal maior ou igual a $5 \mathrm{~kg} / \mathrm{m}^{2}$ em relação ao menor índice atingido. Foi considerado como falha na perda de peso póscirurgia bariátrica quando, decorridos dezoito meses, a perda de peso foi inferior a $50 \%$ do excesso de peso. Reganho seria a recuperação do peso se constatada a perda satisfatória de peso. ${ }^{31}$

Magro e colaboradores discutem a questão do reganho de peso e relatam a manutenção de perda significativa de peso durante os primeiros cinco anos depois do procedimento, principalmente da realização de bypass gástrico em Y de Roux. Acreditase que exista influência relevante no seguimento com equipe multidisciplinar e multiprofissional para que ocorra manutenção da perda de peso bem como da remissão de comorbidades. Além disso, observouse que dezoito meses ou mais seriam o marco do menor valor de índice de massa corporal e da maior perda do excesso de peso. A partir de 24 meses, ocorreu reganho de peso, o qual foi mais pronunciado decorridos 72 meses. $^{32}$
Esses dados são concordantes com o estudo de Adams e colaboradores, que realizaram estudo prospectivo de doze anos. Acompanharam pacientes com comorbidades como diabetes melito do tipo 2 e correlatas e houve taxa de remissão de $75 \%$ e $62 \%$ transcorridos dois e seis anos depois do procedimento cirúrgico respectivamente. Além disso, constatou-se menor reincidência de condições como hipertensão arterial e dislipidemias. Por esse estudo, os pacientes tiveram média de $28 \%$ de perda do peso em seis anos no período pós-cirúrgico. ${ }^{4}$

Comprovadamente, a cirurgia bariátrica é um procedimento que interfere na morbimortalidade por alteração do curso natural de condições como hipertensão, diabetes e dislipidemia em pacientes obesos. 0 procedimento tem um resultado global positivo no diabetes melito do tipo 2 , com número substancial de remissão em pacientes com a doença.

No estudo Stampede, com amostra de 134 pacientes, transcorridos cinco anos depois de realizada a cirurgia bariátrica, observou-se haver maior tendência de os indivíduos diabéticos atingirem e manterem o teor de hemoglobina glicada menor ou igual a $6 \%$ em relação àqueles tratados apenas com terapia medicamentosa para obesidade, a saber, $29 \%$ no grupo com bypass gástrico, $23 \%$ no grupo gastrectomia sleeve e $5 \%$ no grupo sob terapia medicamentosa. Não obstante, ainda se detectou que esses pacientes conseguiram tal controle com menor uso de medicamentos voltados para o controle glicêmico. ${ }^{20}$

Concomitantemente, o estudo Stampede mostra que a cirurgia bariátrica é uma opção no manejo do paciente com obesidade e diabetes, com resultados melhores quando comparados aos da conduta farmacoterapêutica. Entretanto, na avaliação em longo prazo, o estudo evidencia que a remissão prolongada do diabetes no grupo cirúrgico propicia taxas menores que $30 \% .{ }^{20}$

Cabe ressaltar que, mesmo se os pacientes pósbariátricos utilizarem menor número de medicamentos para doenças metabólicas como diabetes e hipertensão arterial, é imperativo acompanhamento e complementação dietética com polivitamínicos, vitamina $B 12$, vitamina $\mathrm{D}$, cálcio e ferro.

Sjöström e colaboradores, no estudo SOS, discutiram a redução do peso e da taxa de mortalidade obtida com a cirurgia bariátrica em comparação com outros métodos. Além disso, também analisaram os efeitos da bariátrica sobre o diabetes, as doenças cardiovasculares e as neoplasias. 0 estudo envolveu a amostra de 2.010 indivíduos obesos, que foram submetidos à cirurgia bariátrica, comparados com 2.037 pacientes obesos, em um grupo de controle, que receberam cuidados habituais. 
Foram realizadas avaliações do peso corporal após dois, dez, quinze e vinte anos. ${ }^{33}$

Comparada com o cuidado usual, a cirurgia bariátrica foi associada com redução em longo prazo da taxa geral de mortalidade. Os dados obtidos demonstraram diminuição da incidência de eventos cardiovasculares, de câncer e de diabetes. Depois de dois anos de seguimento, $72 \%$ de pacientes estavam em remissão do diabetes no grupo de cirurgia. Também foi avaliado o efeito da cirurgia bariátrica na prevenção do diabetes. Em comparação com o cuidado usual, o procedimento reduziu o risco de desenvolvimento de diabetes em $96 \%$, $84 \%$ e $78 \%$ dos pacientes após dois, dez e quinze anos respectivamente. ${ }^{33}$

Recentemente, a Diabetes Surgery Summit (DSS) incluiu a cirurgia metabólica em seu algoritmo de tratamento do diabetes melito do tipo 2 . Acredita-se que a resistência insulínica se desenvolva somente quando a capacidade de armazenamento da gordura subcutânea excede a deposição de gordura ectópica em órgãos como músculo esquelético, fígado, coração, pâncreas e outros. Embora seja altamente relevante a discussão trazida por esse consenso e por estudos apresentados, a recomendação de cirurgia metabólica para pacientes com diabetes melito do tipo 2 não controlado e índice de massa corporal de $30 \mathrm{a}$ $35 \mathrm{~kg} / \mathrm{m}^{2}$ ainda carece de evidências suficientes diante da escassez de estudos prospectivos randomizados a esse respeito. ${ }^{34}$

Estudos recentes com inclusão de pacientes lipodistróficos mostram que a diminuição da capacidade de armazenamento adiposo nos membros inferiores prevê resistência à insulina. ${ }^{35,36}$ Pories e colaboradores trazem um estudo de referência acerca da cirurgia metabólica em pacientes com índice médio de massa corporal de $49,7 \mathrm{~kg} / \mathrm{m}^{2}$, que mostra uma taxa de remissão de $88 \%$ e diminuição rápida dos teores de glicose com bypass gástrico. ${ }^{37}$

Diversos modelos foram propostos para explicar e teorizar a cirurgia bariátrica como instrumento de perda de peso. Ela promove alterações anatômicas dignas de nota e se correlaciona com melhora da qualidade de vida por promover mudanças no estilo de vida ao interferir no metabolismo, na dieta, na prática de atividades físicas e na saúde mental. ${ }^{38}$

Com a perda de peso pós-operatória percebe-se mudança adaptativa na termogênese, apontada como principal fator metabólico envolvido com a cirurgia bariátrica. A redução gradual do gasto energético por diminuição da termogênese modifica a massa gordurosa e muscular e interfere positivamente no processo de manutenção da perda de peso. ${ }^{38}$
Sabe-se que, no período pós-operatório imediato, há redução do consumo calórico induzido pela própria operação. No longo prazo, todavia, a ingesta calórica aumenta gradualmente. Isso se encontra sobremodo associado a qualidade alimentar reduzida, escolhas alimentares inadequadas e falta de acompanhamento nutricional, o que pode estar correlacionado com possivel reganho de peso no período pós-operatório. ${ }^{39}$ Soma-se a isso o fato de que, no contexto do tratamento por via cirúrgica, candidatos à cirurgia bariátrica levam mais tempo para serem saciados e apresentam reflexo de salivação mais lento aos estímulos alimentares e gustativos..$^{40}$

A cirurgia bariátrica também parece desencadear mudança no comportamento alimentar, particularmente em resposta a refeições doces e gordurosas que se tornam menos agradáveis, o que facilita a adoção de alimentação mais saudável e perda de peso subsequente. ${ }^{41}$

Observa-se que o exercício físico de intensidade moderada, isto é, de trinta a quarenta minutos por dia, três a quatro vezes por semana, estaria associado a menor propensão ao reganho de peso, o que ratifica os dados referentes aos hábitos de vida saudáveis adquiridos após cirurgia bariátrica. ${ }^{20,25}$ A modificação de hábitos e sua posterior manutenção, associadas às mudanças fisiológicas e comportamentais relacionadas com o procedimento, resultam em manutenção da perda de peso na fase pós-operatória. ${ }^{21}$

Yanos e colaboradores realizaram um estudo que analisou a influência de alguns fatores na perda de peso e no possivel reganho durante o tempo pósoperatório. Como resultado, observou-se que a maior parte dos fatores encontrados era modificável, o que embasa o princípio de que o seguimento adequado com orientação dietética, atividade física, restrição de substâncias danosas como o álcool em excesso e controle da adesão às modificações de estilo de vida tem grande impacto na manutenção da perda de peso depois do procedimento cirúrgico. ${ }^{42}$

Deve-se ressaltar como fator associado para reganho de peso, além dos fatores supracitados, o grande aumento no consumo de álcool pelos indivíduos submetidos ao procedimento. ${ }^{38}$ Há maior risco - cerca de duas vezes - em relação a pacientes não operados, de aumento do hábito etílico, tendo como hipótese para esse evento a alteração da farmacocinética da substância, que aumenta sua concentração e sensibilidade no organismo e favorece essa desordem psiquiátrica. ${ }^{43}$

Alvarez e colaboradores avaliaram mecanismos que 
poderiam estar associados com o reganho de peso. Concluiu que este estaria relacionado com o volume gástrico retirado cirurgicamente, e volumes inferiores a $500 \mathrm{~mL}$ tiveram maior associação com reganho de peso nos procedimentos do tipo sleeve. 0 autor ressalta, ainda, que é essencial o reconhecimento dos mecanismos envolvidos e a detecção precoce de fatores modificáveis que possam causar o reganho antes que ele se instale e comprometa o resultado cirúrgico. ${ }^{44}$

\section{CONCLUSÃO}

Em face dos dados da literatura científica, a cirurgia bariátrica é uma ótima opção para manejo dos quadros graves de obesidade e refratários ao tratamento medicamentoso. Oferece dados consistentes quanto à perda de peso e ao controle de doenças metabólicas como diabetes, hipertensão e dislipidemia. É fundamental o seguimento adequado, bem como o acompanhamento por equipe multidisciplinar e multiprofissional desses pacientes, pois se observa reganho de peso e recidiva de doenças metabólicas de forma crescente a partir de 24 a 48 meses de transcurso pós-operatório.

\section{REFERÊNCIAS}

1. Polikandrioti M, Stefanou E. Obesity disease. Health Sci J. 2009;3(3):132-8.

2. CDC. Centers for Disease Control and Prevention. Overweight \& Obesity. [acesso 1-8-2019]. Disponivel em: https://www. cdc.gov/obesity/adult/index.html.

3. WHO. World Health Organization. Healthy topics: Obesity. 2018. [acesso 1.0-8-2019]. Disponivel em: http://www.who. int/topics/obesity/en/\&gt.

4. Adams KF, Schatzkin A, Harris TB, Kipnis V, Mouw T, BallardBarbash $\mathrm{R}$ et al. Overweight, obesity, and mortality in a large prospective cohort of persons 50 to 71 years old. N Engl J Med. 2006;355(8):763-78.

5. Prospective Studies Collaboration; Whitlock G, Lewington S, Sherliker P, Clarke R, Emberson J, Halsey J et al. Bodymass index and cause-specific mortality in 900000 adults: collaborative analyses of 57 prospective studies. Lancet. 2009;373(9669):1083-96. DOI: 10.1016/S01406736(09)60318-4.

6. Fontaine KR, Redden DT, Wang C, Westfall AO, Allison DB. Years of life lost due to obesity. JAMA. 2003;289(2):187-93.

7. Finkelstein EA, Khavjou OA, Thompson H, Trogdon JG, Pan L, Sherry $B$ et al. Obesity and severe obesity forecasts through 2030. A J Prev Med. 2012;42(6):563-70. DOI: 10.1016/j. amepre.2011.10.026.

8. Wang $Y$, Beydoun MA, Liang L, Caballero B, Kumanyika SK. Will all Americans become overweight or obese? Estimating the progression and cost of the US obesity epidemic. Obesity (Silver Spring). 2008;16(10):2323-30. DOI: 10.1038/ oby.2008.351.

9. Finucane MM, Stevens GA, Cowan MJ, Danaei G, Lin JK, Paciorek
CJ et al. National, regional, and global trends in body-mass index since 1980: systematic analysis of health examination surveys and epidemiological studies with 960 country-years and 9.1 million participants. Lancet. 2011;377(9765):557-67. DOI: 10.1016/S0140-6736(10)62037-5.

10. Sjöström LV. Mortality of severely obese subjects. Am J Clin Nutr. 1992;55(2Suppl):516S-523S. DOI: 10.1093/ ajcn/55.2.516s.

11. Vigitel Brasil 2017. Vigilância de fatores de risco e proteção para doenças crônicas por inquérito telefônico: estimativas sobre frequência e distribuição sociodemográfica de fatores de risco e proteção para doenças crônicas nas capitais dos 26 estados brasileiros e no Distrito Federal em 2017/Ministério da Saúde, Secretaria de Vigilância em Saúde, Departamento de Vigilância de Doenças e Agravos não Transmissíveis e Promoção da Saúde. Brasília: Ministério da Saúde; 2018.

12. Apovian CM. Obesity: definition, comorbidities, causes, and burden. Am J Manag Care. 2016;22(7 Suppl):s176-85.

13. ABESO. Associação Brasileira para o Estudo da Obesidade e da Síndrome Metabólica. Diretrizes Brasileiras de Obesidade 2016. $4^{\mathrm{a}}$. ed. São Paulo; 2016.

14. Buchwald $H$, Avidor $Y$, Braunwald $E$, Jensen MD, Pories W, Fahrbach $\mathrm{K}$ et al. Bariatric surgery: a systematic review and meta-analysis. JAMA. 2004;292(14):1724-37.

15. Wirth $A$, Wabitsch $M$, Hauner $H$. The prevention and treatment of obesity. Dtsch Arztebl Int. 2014;111(42):705-13. DOI: 10.3238/arztebl.2014.0705.

16. Park JY, Kim YJ. Laparoscopic gastric bypass vs sleeve gastrectomy in obese Korean patients. World J Gastroenterol. 2015;21(44):12612-9. DOI: 10.3748/wjg.v21.i44.12612.

17. Crémieux PY, Ledoux S, Clerici C, Cremieux F, Buessing M. The impact of bariatric surgery on comorbidities and medication use among obese patients. Obes Surg. 2010;20(7):861-70. DOI: $10.1007 / \mathrm{s} 11695-010-0163-6$.

18. Ferraz AA, de Siqueira LT, Nunes Filho E, de Araújo JG Jr, Campos $J M$, de Barros-Correia TX et al. Revision surgery for treatment of weight regain after Roux-en-Y gastric bypass. Obes Surg. 2014;24(1):2-8. DOI: 10.1007/s11695-013-1055-3.

19. de Mattos Zeve JL, Novais PO, de Oliveira Júnior N. Técnicas em cirurgia bariátrica: uma revisão da literatura. Ciência Saúde. 2012;5(2):132-40.

20. Chang SH, Stoll CR, Song J, Varela, JE, Eagon CJ, Colditz GA. The effectiveness and risks of bariatric surgery: an updated systematic review and meta-analysis, 2003-2012. JAMA Surg. 2014;149(3):275-87. DOI: 10.1001/jamasurg.2013.3654.

21. Gloy VL, Briel M, Bhatt DL, Kashyap SR, Schauer PR, Mingrone $G$ et al. Bariatric surgery versus non-surgical treatment for obesity: a systematic review and meta-analysis of randomised controlled trials. BMJ. 2013;347(7931):f5934. DOI: 10.1136/bmj.f5934.

22. Sinclair $P$, Docherty $N$, le Roux CW. Metabolic effects of bariatric surgery. Clin Chem. 2018;64(1):72-81. DOI: 10.1373/ clinchem.2017.272336.

23. Brandao I, Ramalho S, Pinto-Bastos A, Arrojado F, Faria G, Calhau $C$ et al. Metabolic profile and psychological variables after bariatric surgery: association with weight outcomes. Eat Weight Disord. 2015;20(4):513-18. DOI: 10.1007/ s40519-015-0199-7. 
24. Nicoletti CF, de Oliveira BAP, de Pinhel MA, Donati $B$, Marchini JS, Salgado Junior $W$ et al. Influence of excess weight loss and weight regain on biochemical indicators during a 4-year follow-up after Roux-en-Y gastric bypass. Obes Surg. 2015;25(2):279-84. DOI: 10.1007/s11695014-1349-0.

25. Karmali S, Brar B, Shi X, Sharma AM, de Gara C, Birch DW. Weight recidivism post-bariatric surgery: a systematic review. Obes Surg. 2013;23(11):1922-33.

26. Carey RM, Whelton PK. Prevention, detection, evaluation, and management of high blood pressure in adults: synopsis of the 2017 American College of Cardiology/American Heart Association Hypertension Guideline. Ann Intern Med. 2018;168(5):351-8. DOI: 10.7326/M17-3203.

27. Buse JB, Caprio S, Cefalu WT, Ceriello A, Del Prato S, Inzucchi SE et al. How do we define cure of diabetes? Diabetes Care. 2009;32(11):2133-5. DOI: 10.2337/dc09-9036.

28. Berti LV, Campos J, Ramos A, Rossi M, Szego T, Cohen R. Position of the SBCBM- nomenclature and definition of outcomes of bariatric and metabolic surgery. Arq Bras Cir Dig. 2015;28(Suppl1):2. DOI: 10.1590/S010267202015005100002.

29. King WC, Hinerman AS, Belle SH, Wahed AS, Courcoulas AP. Comparison of the performance of common measures of weight regain after bariatric surgery for association with clinical outcomes. JAMA. 2018;320(15):1560-9. DOI: 10.1001/jama.2018.14433.

30. Belle SH, Berk PD, Chapman WH, Christian NJ, Courcoulas AP, Dakin GF et al. Baseline characteristics of participants in the Longitudinal Assessment of Bariatric Surgery-2 (LABS2) study. Surg Obes Relat Dis. 2013;9(6):926-35. DOI: 10.1016/j.soard.2013.01.023.

31. Nedelcu M, Khwaja HA, Rogula TG. Weight regain after bariatric surgery - how should it be defined? Surg Obes Relat Dis. 2016;12(5):1129-30. DOI: 10.1016/j. soard.2016.04.028.

32. Magro DO, Geloneze B, Delfini R, Pareja BC, Callejas F, Pareja JC. Long-term weight regain after gastric bypass: a 5-year prospective study. Obes Surg. 2008;18(6):648-51. DOI: 10.1007/s11695-007-9265-1.

33. Sjöström CD, Lystig T, Lindroos AK. Impact of weight change, secular trends and ageing on cardiovascular risk factors: 10-year experiences from the SOS study. Int J Obes. 2011;35(11):1413-20. DOI: 10.1038/ijo.2010.282.

34. O'Rahilly S. Harveian Oration 2016: Some observations on the causes and consequences of obesity. Clin Med (Lond).
2016;16(6):551-64.

35. Lotta LA, Gulati P, Day FR, Payne F, Ongen H, van de Bunt $M$ et al. Integrative genomic analysis implicates limited peripheral adipose storage capacity in the pathogenesis of human insulin resistance. Nat Genet. 2017;49(1):17-26. DOI: 10.1038/ng.3714.

36. Karpe F, Pinnick KE. Biology of upper-body and lowerbody adipose tissue - link to whole-body phenotypes. Nat Rev Endocrinol. 2015;11(2):90-100. DOI: 10.1038/ nrendo.2014.185.

37. Pories WJ, Swanson MS, MacDonald KG, Long SB, Morris PG, Brown BM et al. Who would have thought it? An operation proves to be the most effective therapy for adult-onset diabetes mellitus. Ann Surg. 1995;222(3):339-52.

38. Cambi MP, Marchesini SD, Baretta GA. Post-bariatric surgery weight regain: evaluation of nutritional profile of candidate patients for endoscopic argon plasma coagulation. Arq Bras Cir Digest. 2015;28(1):40-3. DOI: 10.1590/S010267202015000100011.

39. Wanderley EN, Ferreira VA. Obesidade: uma perspectiva plural. Ciênc Saúde Coletiva. 2010;15(1):185-94. DOl: 10.1590/ S1413-81232010000100024.

40. Marchesi F, De Sario G, Reggiani V, Tartamella F, Giammaresi $A$, Cecchini $S$ et al. Road running after gastric bypass for morbid obesity: rationale and results of a new protocol. Obes Surg. 2015;25(7):1162-70. DOI: 10.1007/s11695-0141517-2.

41. Kalarchian MA, Marcus MD, Courcoulas AP, Cheng Y, Levine $M D$, Josbeno D. Optimizing long-term weight control after bariatric surgery: a pilot study. Surg Obes Relat Dis. 2012;8(6):710-5. DOI: 10.1016/j.soard.2011.04.231.

42. Yanos BR, Saules KK, Schuh LM, Sogg S. Predictors of lowest weight and long-term weight regain among Roux-en-Y gastric bypass patients. Obes Surg. 2015;25(8):1364-70. DOI: 10.1007/s11695-014-1536-z.

43. Azam H, Shahrestani S, Phan K. Alcohol use disorders before and after bariatric surgery: a systematic review and metaanalysis. Ann Transl Med. 2018;6(8):148-56. DOI: 10.21037/ atm.2018.03.16.

44. Alvarez V, Carrasco F, Cuevas A, Valenzuela B, Muñoz G, Guiardo $D$ et al. Mechanisms of long-term weight regain in patients undergoing sleeve gastrectomy. Nutrition. 2015;32(3):303-8. DOI: 10.1016/j.nut.2015.08.023. 